\title{
On Nonnegative Solutions of Random Systems of Linear Inequalities
}

\section{Christian Buchta*}

Mathematisches Institut der Universität, Albertstrasse 23b, D-7800 Freiburg in Breisgau, Federal Republic of Germany

\begin{abstract}
The set of nonnegative solutions of a system of linear equations or inequalities is a convex polyhedron. If the coefficients of the system are chosen at random, the number of vertices of this polyhedron is a random variable. Its expected value, dependent on the probability distribution of the coefficients, which are assumed to be nonnegative throughout, is investigated, and a distribution-independent upper bound for this expected value is established.
\end{abstract}

\section{Introduction}

The number $v$ of vertices of the polyhedron consisting of all points $\left(x_{1}, \ldots, x_{n}\right)$ which satisfy either

$$
\left.\begin{array}{c}
a_{11} x_{1}+\cdots+a_{1 n} x_{n}=b_{1} \\
\cdots \cdots \cdots+\cdots+\cdots+a_{m n} x_{n}=b_{m} \\
a_{m 1} x_{1}+\cdots+
\end{array}\right\} x_{1} \geq 0, \ldots, x_{n} \geq 0
$$

or

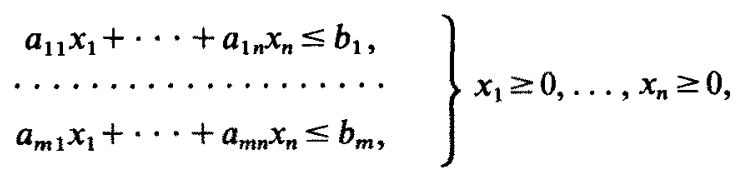

is of great interest in linear programming. Of particular interest is the expected value $E(v)$ of this number if the coefficients in (1) and (2) are random variables.

\footnotetext{
* Permanent address: Institut fïr Analysis, Technische Mathematik und Versicherungsmathematik, Technische Universität, Wiedner Hauptstrasse 8-10, A-1040 Wien, Austria.
} 
From the point of view of applications, the case where these random variables are nonnegative is the most important.

Prékopa [4] determined $E(v)$ if $m=3$ and the coefficients $a_{i j}(i=1,2,3 ; j=$ $1, \ldots, n)$ are independently and uniformly distributed in some interval $[0, a], a>$ 0 , whereas $b_{1}, b_{2}$, and $b_{3}$ have the same fixed value $b>0$. We extend this result in Section 3: for arbitrary $m$, we derive $E(v)$ if the coefficients $a_{i j}(i=1, \ldots, m ; j=$ $1, \ldots, n)$ are distributed independently, continuously, and symmetrically with respect to the same number $c>0$.

A distribution-independent upper bound for $E(v)$ is established in Section 4 for $m \leq 3$. Symmetrically distributed coefficients provide an example where this bound is attained. The main tool in the proof is the knowledge of the probability that the convex hull of three points sampled from a continuous distribution in the plane contains a fixed origin. As this probability is of some interest elsewhere (see Jewell and Romano [3]), we deduce it for any number $s$ of randomly chosen points.

Prékopa [4] derived an upper bound for $E(v)$ in the case where the coefficients $a_{i j}(i=1, \ldots, m ; j=1, \ldots, n)$ and $b_{i}(i=1, \ldots, m)$ are all independently and exponentially distributed with the same parameter. We improve this upper bound in Section 5 and add a lower bound which is close to the improved upper one.

It is probably useful for readers of Prékopa's paper to know that the tedious proofs of Theorems 7 and 8 in his work can be considerably abbreviated by an elegant approach due to Halász and Kleitman [2].

\section{Preliminaries}

In this section we briefly recall some elementary facts which constitute the basis of the following investigations.

Let us assume that all $m \times m$ submatrices of the matrix

$$
\left(\begin{array}{ccc}
a_{11} & \cdots & a_{1 n} \\
\cdots & \cdots & \cdots \\
a_{m 1} & \cdots & a_{m n}
\end{array}\right)
$$

are regular. There are $\left(\begin{array}{c}n \\ m\end{array}\right)$ possibilities of choosing $m$ indices $\left\{j_{1}, \ldots, j_{m}\right\}$ out of $\{1, \ldots, n\}$. To each choice there corresponds a system

$$
\begin{gathered}
a_{1 j_{1}} x_{j_{1}}+\cdots+a_{1 j_{m}} x_{j_{m}}=b_{1}, \\
\cdots \cdots+a_{m j_{m}} x_{j_{m}}=b_{m}, \\
a_{m j_{1}} x_{j_{1}}+\cdots+
\end{gathered}
$$

which has a unique solution $\left(x_{j_{1}}, \ldots, x_{j_{m}}\right)$. The point $\left(x_{1}, \ldots, x_{n}\right)$, where

$$
x_{j}= \begin{cases}x_{j_{k}} & \text { if } j_{k} \in\left\{j_{1}, \ldots, j_{m}\right\}, \\ 0 & \text { else }\end{cases}
$$


is a vertex of polyhedron (1) if all its coordinates are nonnegative. All vertices of polyhedron (1) are obtained on considering all $\left(\begin{array}{c}n \\ m\end{array}\right)$ systems.

Let us also assume that all $m \times m$ submatrices of the matrix

$$
\left(\begin{array}{ccccccc}
a_{11} & \cdots & a_{1 n} & 1 & 0 & \cdots & 0 \\
a_{21} & \cdots & a_{2 n} & 0 & 1 & \cdots & 0 \\
\cdots & \cdots & \ldots & & & & \\
a_{m 1} & \cdots & a_{m n} & 0 & 0 & \cdots & 1
\end{array}\right)
$$

are regular. Introducing slack variables $y_{1}, \ldots, y_{m}$, system (2) can be transformed into a system of type (1):

$$
\left.\begin{array}{c}
a_{11} x_{1}+\cdots+a_{1 n} x_{n}+y_{1}=b_{1}, \\
\cdots \cdots \cdots+\cdots \cdots \cdots+a_{m n} x_{n}+y_{m}=b_{m} \\
a_{m 1} x_{1}+\cdots+a_{1}
\end{array}\right\} \quad x_{1} \geq 0, \ldots, x_{n} \geq 0, \quad y_{1} \geq 0, \ldots, y_{m} \geq 0
$$

Now $\left(\begin{array}{c}n+m \\ m\end{array}\right)$ systems of linear equations have to be considered in order to obtain the vertices of polyhedron $\left(1^{\prime}\right)$, and it is easy to see that to each vertex of polyhedron (1') there corresponds exactly one vertex of polyhedron (2).

We shall be interested in systems (1) and (2) where the coefficients $a_{i j}(i=$ $1, \ldots, m ; j=1, \ldots, n)$ are distributed such that all the mentioned $m \times m$ submatrices have nonvanishing determinants with probability 1 .

If all solutions of the $\left(\begin{array}{c}n \\ m\end{array}\right)$ systems of linear equations (4), corresponding to the $\left(\begin{array}{l}n \\ m\end{array}\right)$ submatrices of $(3)$, have nonnegative coordinates with the same probability, we shall denote this probability by $p_{0}$. Clearly, the expected number $E(v)$ of vertices of polyhedron (1) then equals $p_{0}\left(\begin{array}{l}n \\ m\end{array}\right)$.

Analogously, if, for all $\left(\begin{array}{c}m \\ r\end{array}\right)\left(\begin{array}{c}n \\ m-r\end{array}\right)$ submatrices of (5) with the same number $r$ of unit vectors, the probability that the solution of the corresponding system of linear equations has nonnegative coordinates is the same, we shall denote it by $p_{r}$. Consequently, the expected number $E(v)$ of vertices of polyhedron (2) is given by

$$
E(v)=\sum_{r=0}^{m} p_{r}\left(\begin{array}{c}
m \\
r
\end{array}\right)\left(\begin{array}{c}
n \\
m-r
\end{array}\right)
$$




\section{Symmetrically Distributed Coefficients}

Theorem 1 (Extended Version of Prékopa's Theorem 9). If the coefficients $a_{i j}(i=1, \ldots, m ; j=1, \ldots, n)$ are nonnegative and distributed independently, continuously, and symmetrically with respect to the same number $c>0$, whereas the numbers $b_{i}(i=1, \ldots, m)$ have the same fixed value $b>0$, then the expected number $E(v)$ of vertices of polyhedron (1) is given by

$$
E(v)=\frac{1}{2^{m-1}}\left(\begin{array}{c}
n \\
m
\end{array}\right)
$$

Remark 1. For $m=3$, Prékopa's result

$$
E(v)=\left(\frac{2}{9}+6 \alpha-72 \beta\right)\left(\begin{array}{l}
n \\
3
\end{array}\right),
$$

where $\alpha$ and $\beta$ are given by definite integrals which were evaluated numerically, is incorrect. Formula (5.35) should be

$$
z=\frac{x}{\sqrt{2}-x / \sqrt{2}}
$$

whence formula (5.34) becomes

$$
F(x)=\frac{1}{6} \frac{x}{2-x}, \quad 0 \leq x \leq 1 .
$$

Thus it is easy to calculate the occurring integrals analytically. We obtain $\alpha=1 / 72$, $\beta=1 / 1296$, and consequently, $E(v)=\frac{1}{4}\left(\begin{array}{l}n \\ 3\end{array}\right)$.

In our proof, all these calculations are avoided.

Proof of Theorem 1. As all coefficients $a_{i j}(i=1, \ldots, m ; j=1, \ldots, n)$ are distributed independently and symmetrically with respect to $c$, the points $\left(a_{1 j}, \ldots, a_{m j}\right)(j=1, \ldots, n)$ are distributed independently and symmetrically with respect to the point $(c, \ldots, c)$.

We project these points orthogonally onto the hyperplane of all $\left(z_{1}, \ldots, z_{m}\right)$ with $z_{1}+\cdots+z_{m}=0$. Any two Borel sets on the hyperplane which are symmetrical with respect to the origin have inverse images under the projection which are symmetrical with respect to the point $(c, \ldots, c)$. Thus the marginal distribution is also centrally symmetric: the projected points are distributed independently, continuously, and symmetrically about the origin. It is well known that the convex hull of $m$ such points in $(m-1)$-space contains the origin with probability $1 / 2^{m-1}$ (cf. Remark 2), and we deduce that the solutions of all $\left(\begin{array}{c}n \\ m\end{array}\right)$ systems of linear equations (4) have nonnegative coordinates with the same probability $p_{0}=1 / 2^{m-1}$. 
Remark 2. Wendel [7] showed by a simple and elegant argument that the convex hull of $s$ points, distributed independently, continuously, and centrally symmetrically in $d$-space, contains the centre with probability $1-C(s, d) / 2^{s}$, where $C(s, d)$ denotes the number of regions into which $d$-space is divided by $s$ hyperplanes in general position through the origin. Extending Steiner's [6] three-dimensional result, Schläfli [5, pp. 209-212] proved that

$$
C(s, d)=2 \sum_{k=0}^{d-1}\left(\begin{array}{c}
s-1 \\
k
\end{array}\right)
$$

However, in the proof of Theorem 1 we need only $C(d+1, d)$, which obviously equals $2^{d+1}-2$, as $d$ hyperplanes divide $d$-space into $2^{d}$ regions, and one additional hyperplane does not divide exactly two of these.

Theorem 2 (Extended Version of Prékopa's Theorem 10). If the coefficients $a_{i j}(i=1, \ldots, m ; j=1, \ldots, n)$ are nonnegative and distributed independently, continuously, and symmetrically with respect to the same number $c>0$, whereas the numbers $b_{i}(i=1, \ldots, m)$ have the same fixed value $b>0$, then the expected number $E(v)$ of vertices of polyhedron (2) is given by

$$
E(v)=\frac{1}{2^{m-1}}\left(\begin{array}{c}
n \\
m
\end{array}\right)+\frac{m}{2^{m-1}}\left(\begin{array}{c}
n \\
m-1
\end{array}\right)+O\left(n^{m-2}\right)
$$

as $n$ tends to infinity.

Remark 3. For $m=3$, Prékopa's result

$$
E(v)=\left(\frac{2}{9}+6 \alpha-72 \beta\right)\left(\begin{array}{l}
n \\
3
\end{array}\right)+3\left(\frac{5}{18}-2 \alpha\right)\left(\begin{array}{l}
n \\
2
\end{array}\right)+n+1
$$

is compatible with our expression if the corrected values $\alpha=1 / 72, \beta=1 / 1296$ are substituted.

Proof of Theorem 2. In addition to the proof of Theorem 1, we note that the convex hull of $s$ points of which one is fixed, whereas the remaining $s-1$ points are distributed independently, continuously, and centrally symmetrically, contains the centre with the same probability as the convex hull of $s$ independently, continuously, and centrally symmetrically distributed points. This can be seen by an argument similar to that given by Wendel, cf. Remark 2 . Thus $p_{1}$ is well defined and equals $1 / 2^{m-1}$. 


\section{A Distribution-Independent Estimate}

For $m \leq 3$, we derive in this section a distribution-independent upper bound for $E(v)$. To this end we need the special case $s=3$ of the following observation:

Lemma. Suppose that a continuous probability distribution in the plane is given and let $P$ be an origin and 0 a fixed direction. Denote by $\lambda_{P}(\alpha)$ the probability that a randomly chosen point lies in the cone

$$
\left\{(x, y) \in R^{2}: x=r \cos \varphi, y=r \sin \varphi, 0 \leq r<\infty, 0 \leq \varphi \leq \alpha\right\}
$$

and put

$$
\mu_{p}(\alpha):= \begin{cases}\lambda_{P}(\alpha)-\lambda_{P}(\alpha+\pi)+1 & \text { if } 0 \leq \alpha \leq \pi \\ \lambda_{P}(\alpha)-\lambda_{P}(\alpha-\pi) & \text { if } \pi \leq \alpha \leq 2 \pi\end{cases}
$$

The probability $\rho(P)$ that the convex hull of s points chosen independently according to the given distribution contains $P$ is then given by

$$
\rho(P)=1-\frac{s}{2} \int_{0}^{2 \pi}\left[\left(\mu_{P}(\alpha)\right)^{s-1}+\left(1-\mu_{p}(\alpha)\right)^{s-1}\right] d \lambda_{P}(\alpha)
$$

Proof. Let us call one of the $s$ random points a critical point if all the other $s-1$ points lie on the same side of the line through this point and the origin $P$. Obviously, for each random point, the probability of being a critical point equals

$$
\int_{0}^{2 \pi}\left[\left(\mu_{P}(\alpha)\right)^{s-1}+\left(1-\mu_{P}(\alpha)\right)^{s-1}\right] d \lambda_{P}(\alpha)
$$

the expected number of critical points is thus $s$ times this integral.

On the other hand, the expected number of critical points, on condition that critical points exist, of course, is two, and the expected number of critical points equals the expected number on this condition multiplied by the probability that the condition occurs. Consequently, there exist critical points with probability

$$
\frac{s}{2} \int_{0}^{2 \pi}\left[\left(\mu_{P}(\alpha)\right)^{s-1}+\left(1-\mu_{P}(\alpha)\right)^{s-1}\right] d \lambda_{P}(\alpha) .
$$

As $\rho(P)$ can be interpreted as the probability that no critical points occur, the lemma is proved.

Remark 4. If $s=3$, the lemma yields

$$
\rho(P)=3 \int_{0}^{2 \pi} \mu_{P}(\alpha)\left(1-\mu_{P}(\alpha)\right) d \lambda_{P}(\alpha)-\frac{1}{2}
$$


This result was established by Halász and Kleitman [2] in the special case that the given probability distribution is the uniform distribution on some bounded domain. For arbitrary $s$, a theorem which is equivalent to our lemma was proved by Jewell and Romano [3, pp. 548-551] by more involved considerations.

Theorem 3. Suppose that $m \leq 3$ and that the points $\left(a_{1 j}, \ldots, a_{m j}\right)(j=1, \ldots, n)$ have nonnegative coordinates and are independently, identically, and continuously distributed, whereas $\left(b_{1}, \ldots, b_{m}\right)$ is a fixed point with positive coordinates, then the expected number $E(v)$ of vertices of polyhedron (1) satisfies

$$
E(v) \leq \frac{1}{2^{m-1}}\left(\begin{array}{c}
n \\
m
\end{array}\right)
$$

Proof. We assume that $m=3$. The case $m=2$ is much easier, and the case $m=1$ is trivial.

Analogously to the proof of Theorem 1, we project the points $\left(a_{1 j}, a_{2 j}, a_{3 j}\right)$ $(j=1, \ldots, n)$ orthogonally onto the plane of all $\left(z_{1}, z_{2}, z_{3}\right)$ with $b_{1} z_{1}+b_{2} z_{2}+$ $b_{3} z_{3}=0$. As above, $p_{0}$ is then given by the probability that the convex hull of the first three of the $n$ projected points contains the origin $P$. By the lemma,

$$
p_{0}=3 \int_{0}^{2 \pi} \mu_{P}(\alpha)\left(1-\mu_{P}(\alpha)\right) d \lambda_{P}(\alpha)-\frac{1}{2}
$$

where $\lambda_{P}(\alpha)$ and $\mu_{P}(\alpha)$ are defined by the marginal distribution with respect to the plane. As $\mu_{P}(\alpha)\left(1-\mu_{P}(\alpha)\right) \leq \frac{1}{4}$ for every $\alpha$, we deduce that $p_{0} \leq \frac{1}{4}$. Equality holds if and only if $\mu_{P}(\alpha) \equiv \frac{1}{2}$, i.e., if each line through $P$, which is contained in the plane, divides the plane into two parts of equal measure.

As an immediate consequence we obtain:

Theorem 4. Suppose that $m \leq 3$ and that the points $\left(a_{1 j}, \ldots, a_{m j}\right)(j=1, \ldots, n)$ have nonnegative coordinates and are independently, identically, and continuously distributed, whereas $\left(b_{1}, \ldots, b_{m}\right)$ is a fixed point with positive coordinates, then the expected number $E(v)$ of vertices of polyhedron (2) satisfies

$$
E(v) \leq \frac{1}{2^{m-1}}\left(\begin{array}{c}
n \\
m
\end{array}\right)+O\left(n^{m-1}\right)
$$

as $n$ tends to infinity.

\section{Exponentially Distributed Coefficients}

Theorem 5 (Extended Version of Prékopa's Theorem 5). If for each $i=1, \ldots, m$ the coefficients $a_{i j}(j=1, \ldots, n)$ and $b_{i}$ are independently and exponentially distributed with the same parameter $\lambda_{i}$, then the expected number $E(v)$ of vertices of polyhedron (1) is given by

$$
E(v)=c_{m}\left(\begin{array}{l}
n \\
m
\end{array}\right)
$$


where $c_{1}=1, c_{2}=\frac{1}{3}, c_{3}=\frac{1}{12}$, and, for $m \geq 4$,

$$
\frac{1}{2^{m-1}}\left(\begin{array}{c}
m \\
m / 2
\end{array}\right)^{m}\left(\begin{array}{c}
m^{2} \\
m^{2} / 2
\end{array}\right)^{-1}<c_{m}<\left(\frac{(m+1) !}{m^{m}(m+1)^{m}}\right)^{1 / 2} \text {. }
$$

(In the case of odd $m$, the binomial coefficients are defined by means of the gamma function.)

Proof. As the case $m=1$ is trivial, we may assume that $m \geq 2$. The Jacobi determinant of the transformation

$$
\begin{aligned}
u_{1} & :=\frac{x_{1}}{\sum_{i=1}^{m} x_{i} / \lambda_{i}} \\
\ldots \ldots \ldots \ldots & \ldots \\
u_{m-1} & :=\frac{x_{m-1}}{\sum_{i=1}^{m} x_{i} / \lambda_{i}} \\
v & :=\sum_{i=1}^{m} x_{i} / \lambda_{i}
\end{aligned}
$$

is given by

$$
\frac{\partial\left(u_{1}, \ldots, u_{m-1}, v\right)}{\partial\left(x_{1}, \ldots, x_{m}\right)}=\frac{1}{\lambda_{m} v^{m-1}}
$$

Correspondingly,

$$
\begin{aligned}
& \frac{1}{\lambda_{1}} e^{-x_{1} / \lambda_{1}} \cdots \frac{1}{\lambda_{m}} e^{-x_{m} / \lambda_{m}} d x_{1} \cdots d x_{m} \\
& \quad=\left((m-1) ! \frac{1}{\lambda_{1}} d u_{1} \cdots \frac{1}{\lambda_{m-1}} d u_{m-1}\right)\left(\frac{1}{(m-1) !} v^{m-1} e^{-v} d v\right)
\end{aligned}
$$

It follows that the points

$$
\left(\frac{a_{1 j}}{\sum_{i=1}^{m} a_{i j} / \lambda_{i}}, \ldots, \frac{a_{m j}}{\sum_{i=1}^{m} a_{i j} / \lambda_{i}}\right) \quad(j=1, \ldots, n)
$$

and

$$
\left(\frac{b_{1}}{\sum_{i=1}^{m} b_{i} / \lambda_{i}}, \ldots, \frac{b_{m}}{\sum_{i=1}^{m} b_{i} / \lambda_{i}}\right)
$$


are uniformly distributed over the $(m-1)$-dimensional simplex of all $\left(z_{1}, \ldots, z_{m}\right)$ with

$$
z_{1} / \lambda_{1}+\cdots+z_{m} / \lambda_{m}=1, \quad z_{1} \geq 0, \ldots, z_{m} \geq 0
$$

Furthermore, each of the points is independent of

and

$$
\sum_{i=1}^{m} a_{i j} / \lambda_{i} \quad(j=1, \ldots, n)
$$

$$
\sum_{i=1}^{m} b_{i} / \lambda_{i}
$$

and these sums have a gamma distribution with parameter $m$. (All this is well known at least if $\lambda_{1}=\cdots=\lambda_{m}=1$, in which case the exponential distribution is a special gamma distribution and the uniform distribution occurring over the simplex is a particular case of the $(m-1)$-variate Dirichlet distribution (see, e.g., Wilks [8, p. 179]). However, the above simple argument is included here for the reader's convenience.)

Given $m+1$ independently and uniformly distributed points in an $(m-1)$ dimensional simplex, for each of them, the probability of being contained in the convex hull of the other $m$ points equals the ratio of the expected volume of this convex hull to the volume of the simplex. We thus see that $p_{0}$ is independent of the parameters $\lambda_{i}(i=1, \ldots, m)$ and is just the expected volume $c_{m}$ of the convex hull of $m$ points chosen independently and uniformly from an $(m-1)$ dimensional simplex of volume 1 . It is well known (see [1,pp.3-4]) that $c_{2}=\frac{1}{3}, c_{3}=\frac{1}{12}$, and

$$
c_{m} \geq \frac{1}{2^{m-1}}\left(\begin{array}{c}
m \\
m / 2
\end{array}\right)^{m}\left(\begin{array}{c}
m^{2} \\
m^{2} / 2
\end{array}\right)^{-1}
$$

where equality only holds for $m=2$. Further, the special case $r=0$ of the argument given in the proof of Theorem 6 shows that

$$
c_{m}<\left(\frac{(m+1) !}{m^{m}(m+1)^{m}}\right)^{1 / 2}
$$

Theorem 6 (Extended Version of Prékopa's Theorem 6). Iffor each $i=1, \ldots, m$ the coefficients $a_{i j}(j=1, \ldots, n)$ and $b_{i}$ are independently and exponentially distributed with the same parameter $\lambda_{i}$, then the expected number $E(v)$ of vertices of polyhedron (1) satisfies

$$
\begin{aligned}
& \frac{1}{2^{m-1}}\left(\begin{array}{c}
m \\
m / 2
\end{array}\right)^{m}\left(\begin{array}{c}
m^{2} \\
m^{2} / 2
\end{array}\right)^{-1}\left(\begin{array}{c}
n \\
m
\end{array}\right)+n+1 \\
& \quad \leq E(v)<\sum_{r=0}^{m-2}\left(\frac{(m-r+1) !}{m^{m-r}(m+1)^{m-r}}\right)^{1 / 2}\left(\begin{array}{c}
m \\
r
\end{array}\right)\left(\begin{array}{c}
n \\
m-r
\end{array}\right)+n+1
\end{aligned}
$$

if $m \geq 2$. 
Remark 5. If $m=1$, obviously $E(v)=n+1$. Furthermore, if $m=2, E(v)=$ $\frac{1}{3}\left(\begin{array}{l}n \\ 2\end{array}\right)+n+1$, and, if $m=3, E(v)=\frac{1}{12}\left(\begin{array}{l}n \\ 3\end{array}\right)+\frac{4}{9}\left(\begin{array}{l}n \\ 2\end{array}\right)+n+1$.

Proof of Theorem 6. From the considerations in the proof of Theorem 5 we see that $E(v)$ is independent of the parameters $\lambda_{i}(i=1, \ldots, m)$. We may therefore assume that $\lambda_{1}=\cdots=\lambda_{m}=1$, i.e., all coefficients are identically distributed. We have to estimate the probabilities $p_{r}(r=0, \ldots, m)$, which may be expressed in the form $p_{r}=E\left(\left|\Delta_{r}\right|\right)$, where

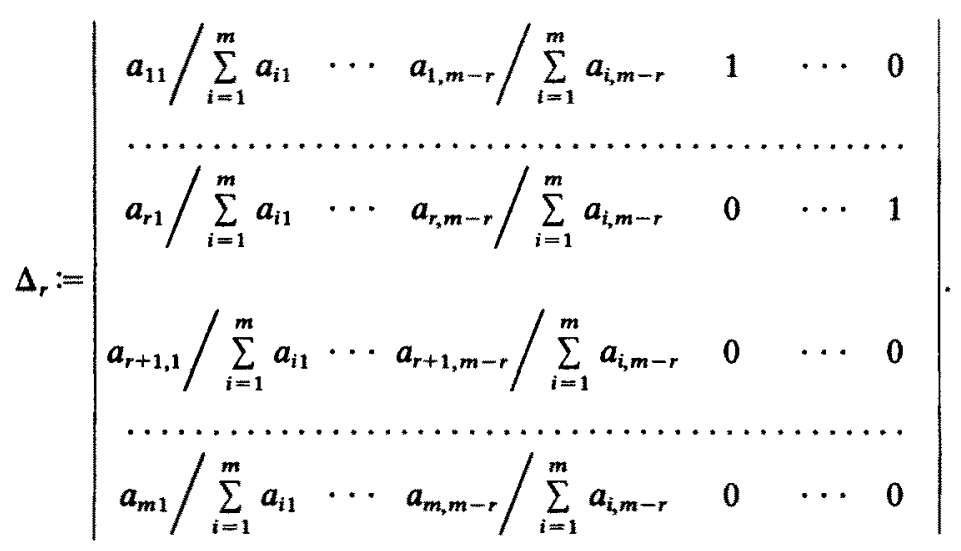

Obviously,

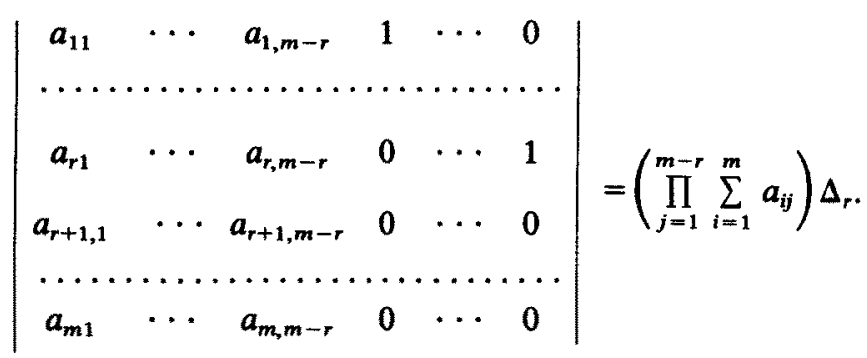

It is well known (see, e.g., Prékopa's Lemma 1) that the second moment of the determinant on the left-hand side equals $(m-r+1)$ !, and it is immediately verified that

$$
E\left(\left(\prod_{j=1}^{m-r} \sum_{i=1}^{m} a_{i j}\right)^{2}\right)=m^{m-r}(m+1)^{m-r}
$$

As the sums

$$
\sum_{i=1}^{m} a_{i j} \quad(j=1, \ldots, n)
$$


are independent of $\Delta_{r}$ (cf. again the proof of Theorem 5), it follows that

$$
E\left(\Delta_{r}^{2}\right)=\frac{(m-r+1) !}{m^{m-r}(m+1)^{m-r}}
$$

whence

$$
p_{r} \leq\left(\frac{(m-r+1) !}{m^{m-r}(m+1)^{m-r}}\right)^{1 / 2} \text {. }
$$

Of course, $p_{m}=1$, and it is easy to see that $p_{m-1}=1 / m$. As $p_{r}>0(r=1, \ldots, m-2)$, we obtain the claimed result.

\section{Acknowledgment}

I am indebted to Professor R. Schneider for most helpful discussions and for carefully reading the manuscript.

\section{References}

1. C. Buchta, Zufällige Polyeder-Eine Übersicht, in Zahlentheoretische Analysis (E. Hlawka, ed.), 1-13, Lecture Notes in Mathematics, Vol. 1114, Springer-Verlag, Berlin-Heidelberg-New YorkTokyo, 1985.

2. S. Halász and D. J. Kleitman, A note on random triangles, Stud. Appl. Math. 53 (1974), 225-237.

3. N. P. Jewell and J. R. Romano, Coverage problems and random convex hulls, J. Appl. Probab. 19 (1982), 546-561.

4. A. Prékopa, On the number of vertices of random convex polyhedra, Period. Math. Hungar. 2 (1972), 259-282.

5. L. Schläfli, Theorie der vielfachen Kontinuität, in Gesammelte Mathematische Abhandlungen, Vol. 1, 167-387, Birkhäuser-Verlag, Basel, 1950.

6. J. Steiner, Einige Gesetze über die Theilung der Ebene und des Raumes, J, Reine Angew. Math. 1 (1826), 349-364.

7. J. G. Wendel, A problem in geometric probability, Math. Scand. 11 (1962), 109-111.

8. S. S. Wilks, Mathematical Statistics, Wiley, New York-London, 1962.

Received October 29, 1985, and in revised form November 8, 1985. 